\title{
Room temperature carbon nanotube based sensor for carbon monoxide detection
}

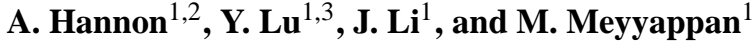 \\ ${ }^{1}$ NASA Ames Research Center, Moffett Field, CA 94035, USA \\ ${ }^{2}$ ERC at NASA Ames Research Center, Moffett Field, CA 94035, USA \\ ${ }^{3}$ ELORET Corporation at NASA Ames Research Center, Moffett Field, CA 94035, USA \\ Correspondence to: M. Meyyappan (m.meyyappan@nasa.gov)
}

Received: 24 July 2014 - Revised: 21 October 2014 - Accepted: 24 November 2014 - Published: 19 December 2014

\begin{abstract}
Sulfonated single-walled carbon nanotubes have been used in an integrated electrode structure for the detection of carbon monoxide. The sensor responds to $0.5 \mathrm{ppm}$ of $\mathrm{CO}$ in air at room temperature. All eight sensors with this material in a 32-sensor array showed good repeatability and reproducibility, with response and recovery times of about $10 \mathrm{~s}$. Pristine nanotubes generally do not respond to carbon monoxide and the results here confirm sulfonated nanotubes to be a potential candidate for the construction of an electronic nose that requires at least a few materials for the selective detection of $\mathrm{CO}$.
\end{abstract}

\section{Introduction}

Carbon monoxide (CO) is an air pollutant and known to have an effect of on global warming. Incomplete burning of coal and hydrocarbon fuels in a variety of applications ranging from power plants to refineries as well as most forms of transport vehicles are the common sources of $\mathrm{CO}$ in the atmosphere. From a safety point of view, it can cause explosion at over $\sim 12 \%$ in air. But as a colorless and odorless gas, $\mathrm{CO}$ poses health risks as well. Upon gaining entry into the lungs through breathing, $\mathrm{CO}$ displaces oxygen leading to suffocation and even death. The recommended exposure limit by the National Institute for Occupational Safety and Health is $50 \mathrm{ppm}$ over an $8 \mathrm{~h}$ work shift. This points to the need for inexpensive and sensitive detection of $\mathrm{CO}$ in the environment, home and office buildings, and other public places.

$\mathrm{CO}$ detection technology has been evolving over a long period and many commercial products are available on the market. Some of the common approaches include electrochemical, catalytic combustion and semiconductor devices. Electrochemical sensors provide selectivity for $\mathrm{CO}$, but their lifetime is limited by the electrolyte. Catalytic combustion based sensors rely on oxidation of $\mathrm{CO}$ and measuring the change in resistance of a metal electrode, but this is typically a high temperature reaction. Recently, novel catalysts that can oxidize at temperatures as low as $70^{\circ} \mathrm{C}$ (Hosaya et al., 2014) have been proposed. The semiconductor type sensors also measure the change in resistance upon adsorption of $\mathrm{CO}$ or any other gas on the surface, and the most common and commercially used technology involves tin oxide thin film based devices (Mishra and Agarwal, 1998). Besides selectivity being an issue, tin oxide and other metal oxide based sensors operate only at elevated temperatures, typically over $200^{\circ} \mathrm{C}$. The nanowire form of the metal oxides has been considered in gas sensor construction in recent years (Meyyappan and Sunkara, 2010) due to the large surface area to volume ratio and other desirable properties. But low or room temperature operation of metal oxide based sensors is not that common, though recently $\mathrm{Pd} / \mathrm{SnO}_{2}$ sensors have been shown to have a good sensitivity in the range of 6-18 ppm of $\mathrm{CO}$ at a temperature of $60^{\circ} \mathrm{C}$ (Kim et al., 2013).

There is a strong push currently to incorporate sensors into smartphones and other mobile devices for environmental monitoring. This incentivizes development of sensors that operate at room temperature and consume low power. In this regard, carbon nanotubes (CNTs) have long been a candidate for room temperature sensing of various gases and vapors; both single-walled and multiwalled CNTs (SWCNTs, MWCNTs) have been extensively studied for gas/vapor sensors (Meyyappan, 2004; Kaufman and Star, 2008; Fam et al., 2011). Typical small molecules participate in charge transfer 
reactions with SWCNTs - either donating an electron to or withdrawing from the nanotubes - which leads to a change in the resistance (or other measureable properties such as capacitance) of the CNTs. SWCNTs have been found to be useful to sense even large molecules (such as nitrotoluene, malathion, etc.) that do not participate in change transfer; in these cases, the molecules can make a conducting bridge between adjacent SWCNTs in an intertube modulation mechanism ( $\mathrm{Li}$ et al., 2003). CO does not participate in a charge transfer process with CNTs according to theoretical and experimental studies (Santucci et al., 2003; Peng and Cho, 2000). However, SWCNTs have been modified, especially with metal loading, to provide a response in the form of a change in resistance upon exposure to $\mathrm{CO}$. Rh-loaded SWCNTs were shown to respond to $2500 \mathrm{ppm}$ of $\mathrm{CO}$ at room temperature (Star et al., 2006). Vertically aligned CNTs decorated with $\mathrm{Pt}, \mathrm{Ru}$ and $\mathrm{Ag}$ clusters responded to $0.1 \% \mathrm{CO}$ at $150{ }^{\circ} \mathrm{C}$ when exposed to a mixture of $\mathrm{CO}, \mathrm{CO}_{2}, \mathrm{NH}_{3}, \mathrm{CH}_{4}$ and $\mathrm{NO}_{2}$, with the mixture representing a landfill gas (Penza et al., 2010). Adding CNTs to an otherwise nonresponsive polyaniline allows CO sensing in the range of 100-1000 ppm at room temperature (Wanna et al., 2006). Similarly, adding CNTs to a mixture of cobalt and tin oxides has also been shown to improve sensor response for $20-1000 \mathrm{ppm}$ of CO, attributed to the ability of nanorubes to increase the adsorption ability of the mixed oxides (Wu et al., 2008).

Other types of modification to CNTs appear to be more promising in terms of room temperature detection of $\mathrm{CO}$ at low concentrations. For example, decorating SWCNTs with a tin oxide nanoparticle enables a $\mathrm{CO}$ detection limit of $1 \mathrm{ppm}$ with a response time of $2 \mathrm{~s}$ and sensitivity of 0.27 for $100 \mathrm{ppm}$. Plasma modification of SWCNTs appears to provide a sensitive response to $5 \mathrm{ppm}$ of $\mathrm{CO}$ at room temperature (Zhao et al., 2012). MWCNTs with the aid of nitrogen doping also show a good response to $\mathrm{CO}$ in the range of 2-20 ppm at ambient and $150^{\circ} \mathrm{C}$ conditions (Adjizian et al., 2014). Here we present $\mathrm{CO}$ detection results using sulfonated SWCNTs with a good response down to $0.5 \mathrm{ppm}$.

\section{Experimental work}

Single-walled carbon nanotubes were purchased from Helix Material Solutions (Richardson, TX) with a purity of $\sim 90 \%$ as claimed by the manufacturer and the sulfonated SWCNTs were provided by South Dakota School of Mines. The nanotubes were first sonicated in concentrated $\mathrm{HNO}_{3}$ for $2 \mathrm{~h}$ and the suspension was refluxed under magnetic stirring at $120^{\circ} \mathrm{C}$ for $2 \mathrm{~h}$. This process helps to remove unreacted catalyst particles while introducing oxygen-containing groups, mainly carboxylic groups, on the SWCNTs (Naseh et al., 2009). The pure SWCNTs were filtered and washed several times, then dried at $120^{\circ} \mathrm{C}$ overnight under vacuum. Then, $25 \mathrm{~mL}$ of concentrated $\mathrm{H}_{2} \mathrm{SO}_{4}$ and $0.25 \mathrm{~g}$ of SWCNTs were mixed and stirred for $5 \mathrm{~h}$ at $300^{\circ} \mathrm{C}$. After cool-
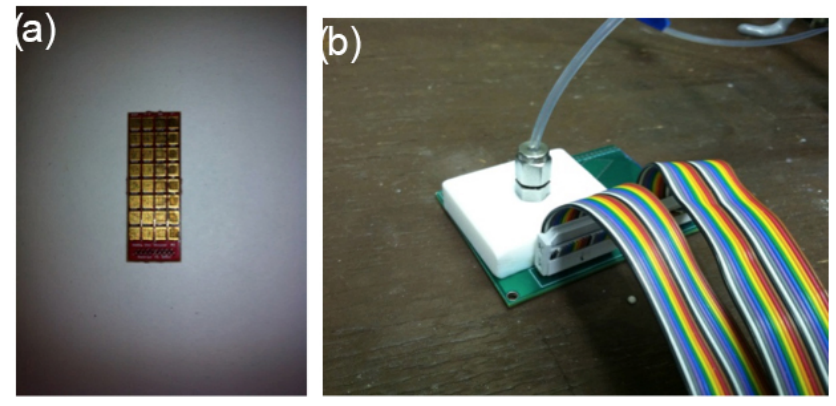

Figure 1. The sensor chip fabricated on a printed circuit board. (a) A 32 sensor array chip and (b) gas exposure unit.

ing down to room temperature, the suspension was diluted with water and filtered. The solids were washed with distilled water to remove excess acid and dried at $100^{\circ} \mathrm{C}$ for $4 \mathrm{~h}$ to obtain sulfonated SWNCTs. This process should lead to the opening of the tube caps and formation of sulfonated groups at defect sites along the sidewalls (Yu et al., 2008). To prepare the solution for sensor preparation, the nanotubes were dispersed ( $0.65 \%$ of nanotubes by weight) in dimethyl formamide (DMF) to form a suspension by sonicating the solution for about $2 \mathrm{~h}$.

Preparation of the interdigitated electrodes on silicon wafers has been described in our previous publications (Lu et al., 2011a, b, c) and a brief account is given below. A printed circuit board (PCB) was used as sensor substrate and a standard photolithography process was used to partially etch away the Au film and define the pattern for the preparation of the sensor chips. The sensor chip consisted of an array of 32 interdigitated electrodes, each with a gap size of $120 \mu \mathrm{m}$. A detailed chip configuration schematic is shown in Fig. 1. The sensing materials ( $0.3 \mu \mathrm{L}$ solution) were deposited on the interdigitated electrode area manually with the aid of a pipette on the chips. Only eight of the 32 sensors were used here and all were coated with the same sulfonated-SWCNT dispersion. Finally, the sensor chip was heated at $80^{\circ} \mathrm{C}$ in air for $1 \mathrm{~h}$ and a thin film of nanotubes bridges across the electrodes after the evaporation of the solvent. The base resistance of the sensors was measured to be in the range of $600 \mathrm{ohm}-$ $5 \mathrm{Kohm}$. The base resistance can be affected by many factors such as the gap size between the interdigitated electrodes, concentration of the nanotubes solution, the amount of solution deposited on the electrode, etc. Indeed, this is the usual range of base resistance that we like to achieve for our sensors, which is normally accomplished by adjusting the solution concentration. Any contribution to base resistance by amorphous carbon can be ruled out, as no amorphous carbon is seen in the SEM image in Fig. 2.

The sensor chip was connected to an interface board through which individual resistances of each sensor channel could be measured. The current-voltage characteristics of each sensor were measured using a Keithley 2700 sys- 


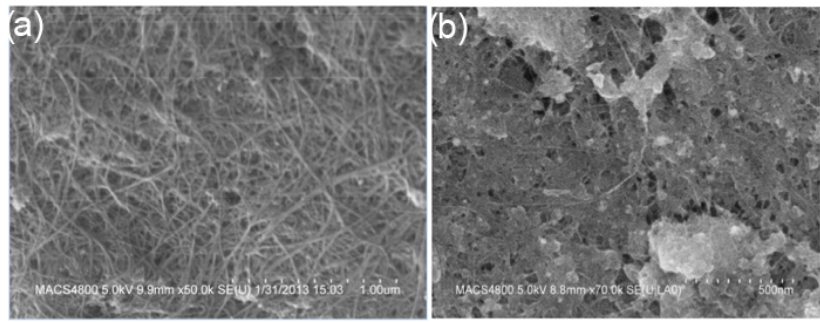

Figure 2. SEM images of (a) pristine and (b) sulfonated singlewalled carbon nanotubes.

tem, and a Environics 2000 gas blending and dilution system was used to create different gas concentration streams. A steady total flow of $400 \mathrm{~cm}^{3} \mathrm{~min}^{-1}$ was used during various gas stream exposures. The sensor chip was exposed to the gas stream using a Teflon cover placed right over the chip to prevent gas leaks as shown in Fig. 1. The sensors were first purged with zero air (Airgas Inc.) for $10 \mathrm{~min}$ to get a stable baseline, and $\mathrm{CO}$ gas exposures were allowed afterwards using a $200 \mathrm{ppm}$ CO balance in air (Airgas Inc.). The sensor tests were performed both in dry air and humid air of $65 \%$ relative humidity $(\mathrm{RH})$.

\section{Results and discussion}

Field emission scanning electron microscopy (FESEM) (Hitachi S-4800 FESEM) and energy-dispersive X-ray spectroscopy (EDX) (Oxford instruments) were used to study the morphological properties and elemental analysis of the nanostructures. The sensing material was deposited on a silicon substrate for obtaining SEM images instead of directly imaging the sensor chip. A comparison of the SWCNTs before and after functionalization reveals that their morphology and structure changed after sulfonation. SWCNTs are covered by a layer of a foreign material, which can be groups of the sulfonic acids (Alamdari et al., 2012). The pristine SWCNT film has a uniform morphology, while the sulfonated SWCNTs appear as thickened bundles of nanotubes and a tangled network to make a cluster as shown in Fig. 2. Some changes in the structural integrity of the nanotubes are observed, caused by the strong acid etching of the nanotubes, which leads to tubes of shorter length. The EDS results (data not shown here) show a sulfur content of $2.72 \%$ (by weight) confirming the presence of sulfonic acid groups. But these sulfonic groups may be superficially on the surface and additional characterization is needed to confirm the nature of functionalization.

Fourier transform infrared spectroscopy (FTIR) spectroscopy was used to investigate the nature of these surface groups as shown in Fig. 3. The spectrum for sulfonated SWCNTs has a number of new peaks not present in the spectrum for pristine nanotubes. For example, a newly observed prominent peak at $1624 \mathrm{~cm}^{-1}$ could be assigned to the $\mathrm{C}=\mathrm{C}$

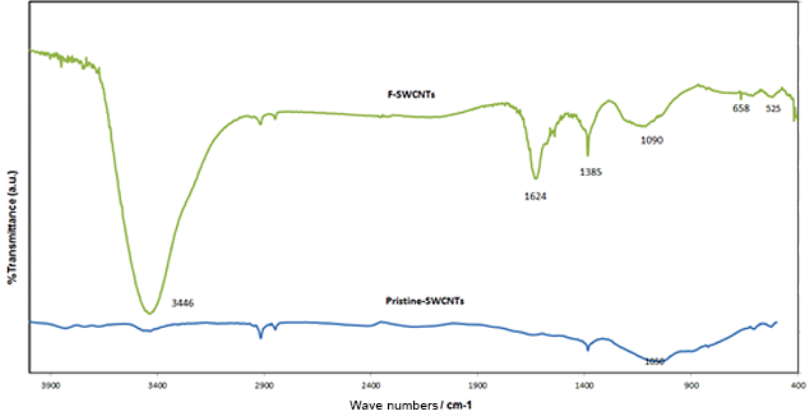

Figure 3. FTIR spectra of pristine and sulfonated SWCNTs.

stretching mode of the SWCNT graphitic layer. This band is weak in pristine SWCNTs due to the symmetry of the dipole moment, but it is intensified with defects on the graphic layer (Yu et al., 2008; Alamdari et al., 2012, 2013). The intensified peak in sulfonated SWCNTs indicates the extensive functionalization of SWCNTs. The presence of carboxylic groups $(\mathrm{C}=\mathrm{O})$ is indicated by the peak at $1718 \mathrm{~cm}^{-1}$. The stretching modes of the sulfate groups in sulfonated nanotubes can be identified with the peaks at 1385 and $1090 \mathrm{~cm}^{-1}$ (Peng et al., 2005). The peak at $658 \mathrm{~cm}^{-1}$ also indicates the $\mathrm{S}=\mathrm{O}$ stretching mode of $-\mathrm{SO}_{3} \mathrm{H}$ (Yu et al., 2008). The sulfonated nanotubes show a very broad peak in the region of 2990 $3700 \mathrm{~cm}^{-1}$, which is assigned to the $\mathrm{O}-\mathrm{H}$ group. The triplet peaks observed at about $2900 \mathrm{~cm}^{-1}$, responsible for the C$\mathrm{H}$ stretching mode, might be the result of hydrocarbon contamination in the spectrometer. In the low-frequency range (see Fig. 3), the peak at $520 \mathrm{~cm}^{-1}$ can be assigned to the $\mathrm{C}-$ $\mathrm{S}$ stretching mode. It is evident from these results that the $\mathrm{H}_{2} \mathrm{SO}_{4}$ treatment enables covalent sulfonation of SWCNTs.

The covalent functionalization was also verified using Raman spectroscopy and Fig. 4 shows the Raman spectra of SWCNTs before and after the acid treatment. There are two major peaks clearly seen at $\sim 1300 \mathrm{~cm}^{-1}$ as the $\mathrm{D}$ band and at $\sim 1590 \mathrm{~cm}^{-1}$ as the $\mathrm{G}$ band. The intensity of the peak at the $\mathrm{D}$ band is due to structural defects or impurities. The $\mathrm{G}$ band indicates the longitudinal stretching vibrations of the sp2 carbons of semiconducting SWCNTs (Yudianti et al., 2011). The ratio of $\mathrm{D}$ and $\mathrm{G}$ peak intensities $\left(\mathrm{I}_{\mathrm{D}} / \mathrm{I}_{\mathrm{G}}\right)$ determines the structure of SWCNTs, and a comparison indicates an increase in the D band after sulfonation due to the strong damage to the sidewalls of SWCNTs or the formation of fragments caused by the functionalization (Yu et al., 2008). The ratio of D / G band intensity shows an increase from 12.5 to $33.21 \%$ after the acid treatment. The observed behavior is more due to the increase in $I_{\mathrm{D}}$ rather than the decrease in $I_{\mathrm{G}}$. The increased $I_{\mathrm{D}} / \mathrm{I}_{\mathrm{G}}$ ratio indicates decreasing symmetry in the structure due to the introduction of functionalized groups and the severe damage to the sidewalls of SWCNTs (Yu et al., 2008). The second-order peak of the D band is observed at $2600 \mathrm{~cm}^{-1}$, called the $\mathrm{G}^{\prime}$ peak. Furthermore, the bands are 


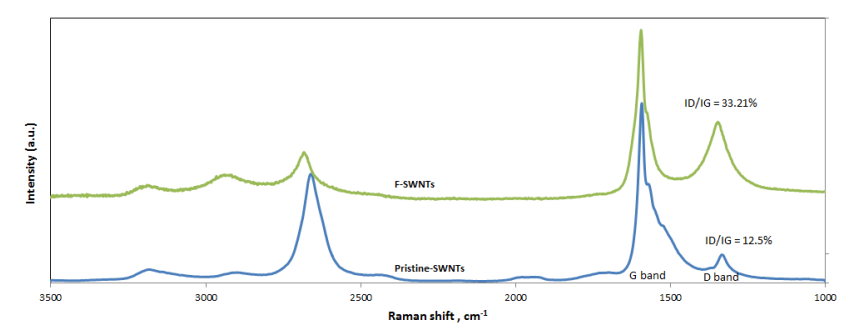

Figure 4. Raman spectra of pristine and sulfonated SWCNTs.

shifted by $10 \mathrm{~cm}^{-1}$ due to the chemical charge transfer on oxidized SWCNTs (Yu et al., 2008).

The sensor chip was exposed to CO dosages of $0.5,2,10$, 25, 50, 60, 75, and $100 \mathrm{ppm}$ at the intervals shown in Fig. 5. The chip was purged with an airflow of $400 \mathrm{~cm}^{3} \mathrm{~min}^{-1}$ for the first $10 \mathrm{~min}$ before any $\mathrm{CO}$ exposure and also in between the $\mathrm{CO}$ exposures. Dry air was used to purge the sensor chip and for $\mathrm{CO}$ dilution to achieve the required concentrations. Figure 5 shows the response curves for the eight sensors. The response plotted here is normalized resistance $\left(R-R_{0}\right) / R_{0}$, where $R_{0}$ is the baseline resistance before gas exposure and $R$ is the resistance at any time $t$ after the gas exposure. A very stable baseline was observed with this material. Signal processing is done here by looking at the relative change in the slope when gas exposure occurs. The response behaviors of all eight sensors are very close to each other. The sensorto-sensor variation is due to the manual deposition process used here for adding the nanotubes to the chip, which results in a variation in nanomaterial density in each sensor element. This can be improved with automated ink-jet-type delivery systems in commercial manufacturing of sensor arrays.

The electrical resistance of the sensor film increases upon exposure to $\mathrm{CO}$. The conductivity change of the sensors is concentration dependent and it increases with concentration in the range of $0.5-50 \mathrm{ppm}$. All eight sensors showed a consistent sensitivity of $0.0014 \pm 0.00015$ as defined by the slope. The sensitivity is defined as the normalized sensor response/ppm of CO concentration. The overall variation in the sensitivity for the sensors is about $<5 \%$, which is similar to common commercial gas/vapor sensors. Pristine SWCNTs did not show any response to CO (data not shown here), while the sulfonated SWCNT sensors showed a clear response to $\mathrm{CO}$ between concentrations of $0.5-50 \mathrm{ppm}$. This behavior is expected since the presence of oxygenated and sulfonated functionalities at the ends of the SWCNTs facilitates electron transfer. The larger response with sulfonated sensors might be the result of the introduction of controlled $\mathrm{COOH}$ and sulfonic acid defects, which form lowenergy adsorption sites and facilitate charge transfer at defect sites. It is hard to state the exact mechanism of the response, as it requires very thorough investigation. At this stage, based on what is known in the literature, we can state the following possibilities for the sensing mechanism.
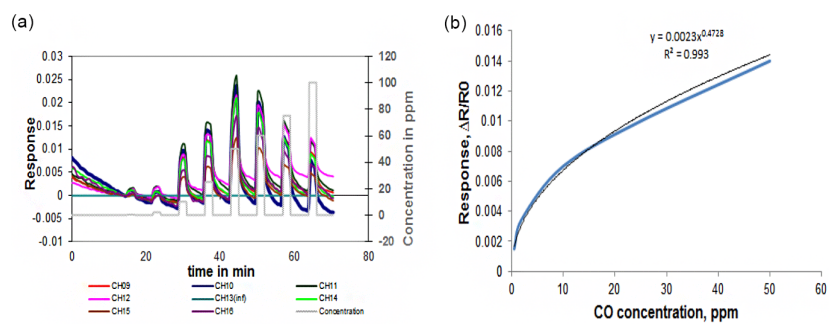

Figure 5. (a) Sensor response to exposure of various dosages of CO and (b) the sensor calibration curve.

$\mathrm{COOH}$-functionalized nanotubes have been widely used as sensing material for sensing $\mathrm{NH}_{3}, \mathrm{Cl}_{2}$, and $\mathrm{CO}$ (Dong et al., 2013; Robinson et al., 2006). As reported by Robinson et al. (2006), the defect sites serve both as low-energy adsorption sites and as nucleation sites for additional condensation of the analyte on the surface of the nanotubes. Sulfonic acids are much stronger acids than the corresponding carboxylic acids. $-\mathrm{SO}_{3} \mathrm{H}$ is also an electron withdrawing group similar to the - $\mathrm{COOH}$ group and, therefore, it is reasonable to consider that the sulfonic acid groups introduced on the surface cause an enhancement of charge density in the SWCNTs; this can increase the amount of electron transfer between sulfonated SWCNT and CO molecules, which increases the hole current of $p$-type sulfonated SWCNT (Dong et al., 2013).

The sensors do not show any concentration dependence at concentrations greater than $50 \mathrm{ppm}$ during the systematic increase in concentration shown in Fig. 5. This might be caused by the limited quantity of the functional groups on the nanotubes. As stated earlier, the $\mathrm{CO}$ response may be due to reducing gas $\mathrm{CO}$ adsorption/interaction at the defect sites; due to the availability of a limited number of defect sites after certain concentration exposure, these sites might be fully occupied. Robinson et al. (2006) reported similar results for acetone and methanol sensing using oxidized nanotubes. The sensors with additional treatment showed better responses due to the introduction of additional defect sites.

The sensor chip was also tested for $\mathrm{CO}$ detection under a $65 \%$ RH environment. The chip was first purged with dry air for $5 \mathrm{~min}$ and then $65 \%$ humidity was introduced for $25 \mathrm{~min}$. The sensor resistance increased significantly in the presence of humidity, but the base resistance returned to the original level after about $20 \mathrm{~min}$. Next, the chip was exposed to 2,10 , $25,50,75$, and $100 \mathrm{ppm}$ of $\mathrm{CO}$ successively at the time interval shown in Fig. 6. All eight sensor channels showed clear responses to $\mathrm{CO}$ and their sensitivity was not significantly reduced. The lack of concentration dependence was seen here as well, but before $50 \mathrm{ppm}$. This might be due to the residual $\mathrm{CO}$ molecules adsorbed on the SWCNT bundles, which are not entirely removed by purging or due to the lack of availability of the active adsorption sites on the nanotube surface. The sensor response and recovery time of about 5-10 s is impressive for room temperature operation. 


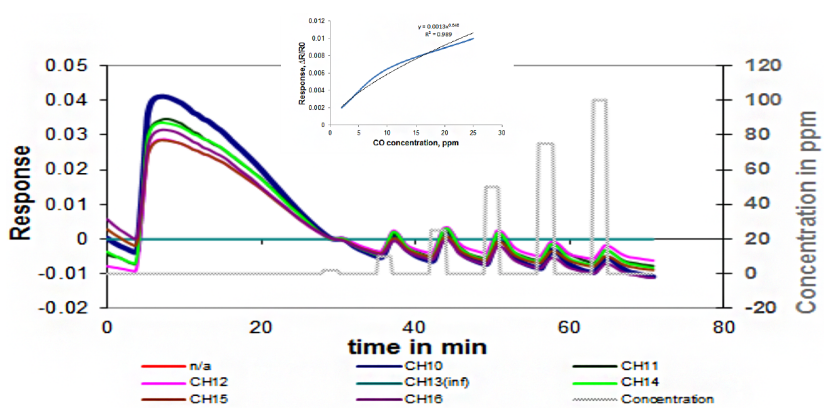

Figure 6. The response of the sensors in Fig. 5 under a $65 \% \mathrm{RH}$ environment.

\section{Conclusions}

Environmental monitoring via smartphones and wearable devices has been gaining popularity, but is in early stages of development. Carbon monoxide is a key pollutant that would be found in any wish list of gases to be monitored. Sensitive detection of $\mathrm{CO}$ at room temperature is critical to construct sensors to meet the needs above. Conventional semiconductor sensors based on oxide conductors typically work at elevated temperatures. In this regard, single-walled carbon nanotubes offer an alternative for room temperature gas/vapor sensing, as has been demonstrated in the literature for numerous analytes. But SWCNTs do not respond to $\mathrm{CO}$ at any temperature. Construction of an electronic nose consisting of a multisensor array for the detection of any analyte requires at least a few different materials, which respond to that analyte. Thus far, tin oxide loading of SWCNTs (Zhang et al., 2013) and oxygen plasma modified nanotubes (Zhao et al., 2012) have been reported to be effective in CO detection. In the present work, we have shown that sulfonated SWCNTs provide a good response to $\mathrm{CO}$ down to $0.5 \mathrm{ppm}$, and thus can be added to the arsenal of responsive materials for the e-nose construction. Future work will include these different materials and report the operation of our current 32-sensor platform as e-nose. The lifetime of the present $\mathrm{CO}$ sensors has not been investigated here, but we have sensors for ammonia, chlorine and $\mathrm{NO}_{2}$, which were made in 2008, still showing sensitivity to these gases. We anticipate a similar lifetime performance for the $\mathrm{CO}$ sensors as well, and this will be studied in the future. Future work also should include efforts to improve the detection limit even further while maintaining high selectivity and possibilities to transfer the approach to flexible substrates including paper (Han et al., 2013, 2014).

Acknowledgements. This work was supported by the Nanotechnology Thematic Project in NASA's Game Changing Development Program. The smartphone development was funded by the US Department of Homeland Security, HSARPA Cell-All program via a NASA-DHS interagency agreement (IAA: HSHQDC-08$\mathrm{X}$-00870). The work conducted by the employees of ERC and ELORET Corporation was supported through subcontracts to the respective organizations. The authors acknowledge H. Hong from South Dakota School of Mines for providing sulfonated SWCNT samples and D. Skiver, M. Shi and A. Nguyen for help with SEM imaging.

Edited by: A. Romano-Rodriguez

Reviewed by: two anonymous referees

\section{References}

Adjizian, J. J., Leghrib, R., Koos, A. A., Suarez-Martinez, I., Crossley, A., Wagner, P., Grobert, N., Liobet, E., and Ewels, C. P.: Boron and nitrogen-doped multiwall carbon nanotubes for gas detection, Carbon, 66, 662-673, 2014.

Almdari, R., Golestanzadeh, M., Agend, F., and Zekti, N.: Sulfonic acid functionlized single walled carbon nanotubes: A highly efficient and reusable catalyst for green synthesis of 14-Aryl-14Hdibenzo[a,j] xanthene derivatives under solvent-free conditions, ICNS4, 2012.

Alamdari, R., Golestanzadeh, M., Agend, F., and Zekri, N.: Synthesis, characterization and catalytic activity of sulphonated multiwalled carbon nanotubes as heterogeneous, robust and reusable catalysts for the synthesis of bisphenolic antioxidants under solvent-free conditions, J. Chem. Sci., 125, 1185-1195, 2013.

Dong, K.-Y., Choi, J., Lee, Y. D., Kang, B. H., Yu, Y-Y., Choi, H. H., and Ju, B.-K.: Detection of a $\mathrm{CO}$ and $\mathrm{NH}_{3}$ gas mixture using carboxylic acid-functionalized single-walled carbon nanotubes, Nanoscale Res. Lett., 8, 12-18, 2013.

Fam, D. W. H., Palaniappan, A. I., Tok, A. I. R., Liedberg, B., and Moochhala, S. M.: A review on technological aspects influencing commercialization of carbon nanotube sensors, Sens. Actuat. B., 157, 1-7, 2011.

Han, J. W., Kim, B., Li, J., and Meyyappan, M.: A carbon nanotube based ammonia sensor on cotton textile, Appl. Phys. Lett., 102, 193104, doi:10.1063/1.4805025, 2013.

Han, J. W., Kim, B., Li, J., and Meyyappan, M.: A carbon nanotube based ammonia sensor on cellulose paper, RSC Adv., 4, 549553, 2014.

Hosaya, A., Tamura, S., and Imanaka, N.: A catalytic combustiontype $\mathrm{CO}$ gas sensor incorporating aluminum nitride as an intermediate heat transfer layer for accelerated response time, J. Sensors Sensor Syst., 3, 141-144, 2014.

Kaufman, D. R. and Star, A.: Carbon nanotube gas and vapor sensors, Angew. Chem. Int. Edit., 47, 6550-6570, 2008.

Kim, B., Lu, Y., Hannon, A., Meyyappan, M., and Li, J.: Low temperature $\mathrm{Pd} / \mathrm{SnO}_{2}$ sensor for carbon monoxide detection, Sens. Actuat. B., 177, 770-775, 2013.

Li, J., Lu, Y., Ye, Q., Cinke, M., Han, J., and Meyyappan, M.: Carbon nanotube sensors for gas and organic vapor detection, Nano Lett., 3, 929-933, 2003.

Lu, Y., Meyyappan, M., and Li, J.: A carbon-nanotube-based sensor Array for formaldehyde detection, Nanotechnology, 22, 055502, doi:10.1088/0957-4484/22/5/055502, 2011a.

Lu, Y., Meyyappan, M., and Li, J.: Fabrication of carbon nanotubebased sensor array and interference study, J. Mater. Res., 26, 2017-2023, 2011b.

Lu, Y., Meyyappan, M., and Li, J.: Trace detection of hydrogen peroxide vapor using carbon-nanotube-based chemical sensor, Small, 7, 1714-1718, 2011c. 
Meyyappan, M. (Ed.): Carbon Nanotubes: Science and Applications, CRC Press, 2004.

Meyyappan, M. and Sunkara, M. K.: Inorganic nanowires: applications, properties and characterization, CRC Press, Boca Raton, FL, See Chapter 14, 2010.

Mishra, V. N. and Agarwal, R. P.: Sensitivity, response and recovery time of $\mathrm{SnO}_{2}$ based think-film sensor array for $\mathrm{H}_{2}, \mathrm{CO}, \mathrm{CH}_{4}$ and LPG, Sens. Actuat. B., 29, 861-874, 1998.

Naseh, M., Khodadadi, A., Mortazavi, Y., Sahraei, O., Pourfayaz, F., and Sedghi, S.: Functionalization of carbon nanotubes using nitric acid oxidation and DBD plasma, World Academy of Science, Eng. Technol., 49, 177-179, 2009

Peng, F., Zhang, L., Wang, H., Lu, P., and Yu, H.: Sulfonated carbon nantotubes as a strong protonic acid catalyst, Carbon, 43, 239782429, 2005.

Peng, S. and Cho, K. J.: Chemical control of nanotube electronics, Nanotechnology, 11, 57-60, 2000.

Penza, M., Rossi, R., Aluisi, M., and Serra, E.: Metal-modified and vertically aligned carbon nanotube sensors array for landfill gas monitoring applications, Nanotechnology, 21, 105501, doi:10.1088/0957-4484/21/10/105501, 2010.

Robinson, J. A., Snow, E. S., Badescu, S. C., Reinecke, T. L., and Perkins, F. K.: Role of defects in single-walled carbon nanotube chemical sensors, Nano Lett., 6, 1747-1751, 2006.

Santucci, S., Picozzi, S., Gregorio, F. D., Lozzi, L., Cantalini, C., Valentini, L., Kenney, J. M., and Delley, B.: $\mathrm{NO}_{2}$ and $\mathrm{CO}$ gas adsoprtion on carbon nanotubes: experiment and theory, J. Chem. Phys., 119, 10904-10910, 2003.
Star, A., Joshi, V., Skarupo, S., Thomas, D., and Gabriel, J. C. P.: Gas sensor array based on metal decorated carbon nanotubes, J. Phys. Chem. B., 110, 21014-21020, 2006.

Wanna, Y., Srisukhumbowornchai, N., Tauntranont, A., Wisitsoraat, N., Thavarungkul, P., and Singjai, P.: The effect of carbon nanotube dispersion on $\mathrm{CO}$ gas sensing characteristics of polyaniline gas sensor, J. Nanosci. Nanotechno., 6, 3893-3896, 2006.

Wu, R. J., Wu, J. G., Yu, M. R., Tsai, T. K., and Yeh, C. T.: Use of $\mathrm{CNT} / \mathrm{CO}_{3} \mathrm{O}_{4}-\mathrm{SnO}_{2}$ in a carbon monoxide sensor operating at room temperatures, Sensor Lett., 6, 848-851, 2008.

Yu, H., Jin, Y., Li, Z., Peng, F., and Wang, H.: Synthesis and characterization of sulfonated single-walled carbon nanotubes and their performance as solid acid catalyst, J. Solid State Chem., 181, 432-438, 2008.

Yudianti, R., Onggo, H., Surdiram, Satio, Y., Iwata, T., and Azuma, J.: Analysis of functional group sited on multi-wall carbon nanotubes surface, Open Mat. Sci. J., 5, 242-247, 2011.

Zhang, Y., Cui, S., Chang, J., Ocola, L. E., and Chen, J.: Highly sensitive room temperature carbon monoxide detection using $\mathrm{SnO}_{2}$ nanoparticle-decorated semiconducting single-walled carbon nanotubes, Nanotechnology, 24, 025503, doi:10.1590/15161439.235713, 2013.

Zhao, W., Fam, D. W. H., Yin, Z., Sun, T., Tan, H. T., Liu, W., Tok, A. L. Y., Boey, Y. C. F., Zhang, H., Hng, H. H., and Yan, Q.: A carbon monoxide gas sensor using oxygen plasma modified carbon nanotubes, Nanotechnology, 23, 425502, doi:10.1088/09574484/23/42/425502, 2012. 